\title{
The 2021 Survey of the Association of Directors of Medical Student Education in Psychiatry
}

\author{
Rachel A. Russo ${ }^{1}$ (D) $\cdot$ Mary C. Blazek ${ }^{2} \cdot$ Lia A. Thomas ${ }^{1}$
}

Received: 29 July 2021 / Accepted: 21 September 2021 / Published online: 29 September 2021

This is a U.S. government work and not under copyright protection in the U.S.; foreign copyright protection may apply 2021

\section{To the Editor:}

The Association of Directors of Medical Student Education in Psychiatry (ADMSEP) was created in 1974 to promote "excellence in behavioral sciences and psychiatric education for medical students" [1]. In support of this mission, the ADMSEP membership has been surveyed approximately every 5 years starting in 2005 to look at trends in medical student education in psychiatry.

In February 2021, an invitation to complete the fourth ADMSEP survey was sent electronically to the 260 members of the organization's listserv. Weekly reminders were sent, and the survey closed after 1 month. The survey was anonymous, and consent was implied with completion of the survey. The University of Michigan Institutional Review Board determined this study was exempt from ongoing review. The survey was developed by the authors on the basis of previous surveys and contained 61 questions, including 14 new questions drafted by ADMSEP's two new task forces, exploring diversity, equity, inclusion, and anti-racism (DEIA) and the effective use of medical student performance evaluations. The authors received 119 unique responses for an estimated response rate of $45.8 \%$. Fifty-one respondents (42.9\%) identified themselves as clerkship directors, with many holding additional titles in their programs. Clerkship directors had spent an average of $6.29( \pm 5.74)$ years in that role.

The ADMSEP survey provides a snapshot into current medical education, which can be compared to data from previous years. Overall, most scaled items were like 2016. Most educators agreed that their role in medical student education and membership in ADMSEP have contributed to

Rachel A. Russo

Rachel.Russo@UTSouthwestern.edu

1 VA North Texas Health Care System and University of Texas - Southwestern Medical Center, Dallas, TX, USA

2 University of Michigan Medical School, Ann Arbor, MI, USA satisfaction in their work and to advancement in their career, consistent with findings reported from larger surveys of core clerkship directors [2]. Respondents indicated they received less adequate mentoring for their role as an educator and less access to research consults and educational design consultants compared to the 2016 survey [3]. Other areas showed no change. Administrative support remained relatively consistent with past surveys, but the amount of time paid by institutions for directing the clerkship has shifted. Compared to 2016, the percent of directors who received $41-50 \%$ effort increased from 21.8 to $28.5 \%$, and the percentage of directors reporting $20 \%$ or less dedicated time increased from 12.7 to $31 \%$ [2].

As to the new questions, respondents were asked how much content in their current clerkship didactic curriculum addresses DEIA: $27.17 \%$ reported $0 \mathrm{~h}, 57.61 \%$ 1-2 h, $14.13 \% 3-5 \mathrm{~h}$, and $1.06 \% 6$ or more $\mathrm{h} ; 72.04 \%$ of respondents reported their institution has DEIA content in the preclerkship. Although Liaison Committee on Medical Education standards dictate that medical school curricula contain the basic principles of culturally competent care, results of this survey highlight that more than $75 \%$ of clerkships contained $2 \mathrm{~h}$ or less of applicable content. Among respondents, $83.87 \%$ reported they would be interested in including a DEIA module in the clerkship if one were developed by ADMSEP. Respondents were asked to identify DEIA topics they felt were of most importance and mentioned structural racism in medicine and psychiatry $(n=13)$, implicit bias $(n=10)$, LGBTQ + mental health $(n=6)$, anti-racism $(n=5)$, and microaggressions $(n=4)$. Survey respondents in general supported the US Medical Licensing Examination Step 1 going to pass/fail, with $57.74 \%$ supporting it and $26.8 \%$ undecided. Respondents were less sure about whether psychiatry should create a standard letter of evaluation, with $45.35 \%$ saying not sure and $27.84 \%$ responding affirmatively. The remaining questions were answered by persons who identified themselves as clerkship directors. When asked whether as clerkship directors they had enough information 
to send a holistic review to program directors, $56 \%$ agreed and $22 \%$ were unsure. Reported barriers preventing a more holistic review of students included need for more detailed or differentiated clinical faculty feedback $(n=10)$, limited time with students $(n=8)$, and not working directly with all students $(n=5)$.

This study had several limitations. Because the overall survey response rate was $45.8 \%$ and only 51 persons identified as clerkship directors, the resulting findings may not be representative of all psychiatry clerkship directors. Also, this survey was administered during the COVID-19 pandemic. Response rate and responses might reflect the impact of lockdown and adjustments to differing restrictions across institutions, regions, or states.

The ADMSEP 2021 survey provides a snapshot of education and administration in undergraduate medical education in psychiatry. Dedicated time for the clerkship director role shifted but, overall, still reflects a predominance of programs with insufficient dedicated effort. The Alliance for Clinical Education has recommended a minimum of $50 \%$ time for the position [4]. At least 65\% of clerkship director respondents fell below this recommendation. Advocacy for more dedicated time for clerkship directors in these programs would support the role and, therefore, medical student education. In this survey, five respondents reported being in the clerkship director position for 1 year or less. COVID-19 has had many impacts on health care educational environments, and it may have increased turnover. A further survey could help clarify this and other changes related to COVID-19. The survey also provides perspective on two topical issues in the forefront of medical education today. More than $75 \%$ of clerkships contained $2 \mathrm{~h}$ or less of applicable DEIA content. It is possible that this topic is covered in other parts of medical student education, but social determinants of health and implicit bias have special impact on mental health, warranting dedicated focus in pre-clinical and clerkship psychiatry curricula [5]. There is also a lack of meaningful assessment methods in medical school provided to program directors, and in our survey, most clerkship directors agreed that they have information appropriate for a standard letter of evaluation and enough information to send a holistic review to program directors. Discussion is needed about developing a standard letter of evaluation for psychiatry or other metrics to assess relevant clinical skills. ADMSEP can provide a venue for development of resources and sharing of best practices on both these issues, and the dissemination of its survey results can help its members advocate for themselves within their institutions.

\section{Declarations}

Ethics Approval The University of Michigan IRB designated this study to be exempt from review.

Disclosures Dr. Lia Thomas is on the editorial board for the journal Academic Psychiatry. Manuscripts that are authored by an editor undergo the same editorial review process applied to all manuscripts, including double-blinded peer review. On behalf of all authors, the corresponding author states that there is no conflict of interest.

\section{References}

1. Association of Directors of Medical Students in Psychiatry. ADMSEP Mission Statement. 2021. https://www.admsep.org/about. php?l=ADMSEP. Accessed 9 Apr 2021.

2. Ephgrave K, Margo KL, White C, Hammoud M, Brodkey A, et al. Core clerkship directors: their current resources and the rewards of the role. Acad Med. 2010;85(4):710-5.

3. Thomas LA, Dallaghan GB, Balon RM. The 2016 survey of the association of directors of medical student education in psychiatry. Acad Psychiatry. 2018;42:366-70.

4. Pangaro L, Bachicha J, Brodkey A, Chumley-Jones H, Fincher RM, Gelb D, et al. Expectations of and for clerkship directors: a collaborative statement from the Alliance for Clinical Education. Teach Learn Med. 2003;15(3):217-22.

5. Sederer LI. The social determinants of mental health. Psychiatr Serv. 2016;67(2):234-5.

Publisher's Note Springer Nature remains neutral with regard to jurisdictional claims in published maps and institutional affiliations. 